\title{
MODIFIED HEURISTIC TIME DEVIATION TECHNIQUE FOR JOB SEQUENCING AND COMPUTATION OF MINIMUM TOTAL ELAPSED TIME
}

\author{
N.Nagamalleswara Rao ${ }^{1}$ Dr. O. Naga Raju ${ }^{2}$ and Prof. I. Ramesh Babu ${ }^{3}$ \\ ${ }^{1}$ Department of C S \& E, R.V.R \& J. C. College of Engineering, Guntur, A. P, India \\ nnmr_meyahoo.com \\ ${ }^{2,3}$ Department of Computer Science and Engineering,Nagarjuna University, \\ Guntur, Andhra Pradesh, India \\ onrajunrt@gmail.com, rinampudi@hotmail.com
}

\begin{abstract}
Job sequencing is the arrangement of the task that is to be performed or processed in a machine in that particular order. Job sequencing problem has become the major problem in the computer field. A finite set of $n$ jobs where each job consists of a chain of operations and a finite set of $m$ machines where each machine can handle at most one operation at a time. Each operation needs to be processed during an. uninterrupted period of a given length on a given machine and our Purpose is to find a schedule, that is, an allocation of the operations to time intervals to machines that has minimal length.
\end{abstract}

\section{KEYWORDS}

Job sequencing, processing time, Schedule, non-preemptive, preemptive, time deviation, time duration, Total Elapsed Time, heuristic technique, sequencing rules, earliest due date, Shortest processing time rule.

\section{JOB SEQUENCING PROBLEM}

Job Sequencing is the arrangement of the tasks required to be carried out sequentially. A set of jobs need to be served by a server which can process only one job at a time. Each job has a finite processing time and a per unit time waiting cost [1]. Each job has a specified processing order through the machines, i.e. a job is composed of an ordered list of operations each of which is determined by the machine required and the processing time on it [2]. Processing times of some operations are represented by a uniform distribution with given lower and upper bounds. The objective is to find a predictive schedule that can deal with this uncertainty [3]. In job sequencing, there may be a finite set of $n$ jobs where each job consists of a chain of operations and it consists of a finite set of $m$ machines. Each machine can handle at most one operation at a time. Each operation needs to be processed during an uninterrupted period of a given length on a given machine. Then find a schedule, that is, an allocation of the operations to time intervals to machines that has minimal length. To schedule several jobs, the intervals selected for the jobs must not overlap. The objective is to schedule as many jobs as possible under the constraints [4].

Job sequences are optionally restartable. If you run a restartable sequence and one of the jobs fails to finish correctly, you can fix the problem, then re-run the sequence from the point at which it left off.. 
In general, scheduling policies may be preemptive or non-preemptive. In a non-preemptive pure multiprogramming system, the short-term scheduler lets the current process run until it blocks, waiting for an event or a resource, or it terminates. Preemptive policies, on the other hand, force the currently active process to release the CPU on certain events, such as a clock interrupt, some $\mathrm{I} / \mathrm{O}$ interrupts, or a system call. Scheduling is significantly investigated in manufacturing systems. Scheduling has been applied to meet customer demand, which plays an important role in customer satisfaction [5].

Sequencing problems arise when there is a choice as to the order in which a number of tasks can be performed. In such problems, we determine an appropriate order or sequence for a series of jobs to be done on a finite number of service facilities [6]. The Job-Shop Scheduling Problem (JSSP) is considered as one of the difficult combinatorial optimization problems and treated as a member of NP-complete problem class [7]. The problem has been known for years in the scheduling theory as a particularly hard combinatorial optimization case [8]. The researcher must be concerned not only with obtaining an optimal solution but also with the practical and economical application of the solution technique [9]. Multi-objective scheduling problems often involve minimizing the makespan while considering setup costs and times [10].

Normally performance can be measured by means of Job flow time: Time a job is completed minus the time the job was first available for processing; avg. flow time measures responsiveness, Average jobs in system: Measures amount of work-in-progress; avg. \# measures responsiveness and work-in-process inventory, Makespan: The time it takes to finish a batch of jobs; measure of efficiency, Job lateness: Whether the job is completed ahead of, on, or behind schedule, Job tardiness: How long after the due date a job was completed, measures due date performance.

Scheduling and sequencing are similar terms. Scheduling provides a detail plan over time. Sequencing does not refer to time at all. Sometimes, a unique schedule follows from a given sequence. In such a case, it's enough to solve the sequencing problem and not worry about the detail scheduling problem. Job sequencing deals with the determination of the appropriate order in which waiting jobs should be served. Due to the combinational nature of sequencing problems, when the number of jobs is very large, the number of alternative job sequences also becomes very large. That is why numerous heuristic procedures have been developed for special case problems. There is several priority sequencing rules (e.g., first in first out, last in first out, shortest processing time first and longest processing time first) used in the assignment of jobs to one machine. A new heuristic technique called Time deviation method will be used to obtain a sequence of jobs for solving job sequencing problem by which the total elapsed time will get reduced.

\section{CLASSIFICATION OF JOB SEQUENCING ALGORITHMS}

There were various algorithms for solving job sequencing problems. Some of the algorithms are explained below.

\subsection{TABU SEARCH ALGORITHM}

Eugeniusz Nowicki and CzeslAw Smutnicki [8] have proposed an advanced tabu search algorithm for the job shop problem. The job shop scheduling problem with the makespan criterion was a certain NP-hard case from OR theory that had excellent practical applications. This problem, had been examined for years, is also regarded as an indicator of the quality of advanced scheduling algorithms. It provided a new approximate algorithm that was based on the big valley phenomenon, and used some elements of so-called path re linking technique as well as new theoretical properties of neighborhoods. The algorithm thus provided a powerful tool to solve the job shop problem with the makespan criterion. It offered very good accuracy, in comparison to 
other best known approaches, obtainable in a short running time on a modern PC. Those properties, confirmed through exhaustive tests on all known benchmarks, followed from the suitable use of properties of the solution space, especially BV. The general idea of the algorithm could be applied to other scheduling problems, as an example, the flow shop and hybrid flow shop problem.

\subsection{FUZZY TOPSIS METHOD}

Pragati Jain and Manisha Jain [6] have presented Fuzzy TOPSIS Method in Job Sequencing Problems on machines of unequal efficiencies. Sequencing problems arise when there was a choice as to the order in which a number of tasks can be performed. In such problems, they determined an appropriate order or sequence for a series of jobs to be done on a finite number of service facilities. They presented the way of making sequence of a finite number of jobs on a finite number of machines of unequal efficiencies by using the technique of order preference by similarity to ideal solution or simply TOPSIS Method in fuzzy environment. The order of machines was random. The time taken by the machines for conducting jobs was assumed as imprecise processing time or fuzzy numbers. The fuzzy evaluation values were given by triangular fuzzy numbers. Weights were given to each machine according to their efficiency. A new distance was defined using which the distance of each job from the positive and negative ideal solutions were calculated. Finally a closeness coefficient was defined and that determined the ranking order of the jobs so that the complete project got finished at minimum time-span. A numerical example demonstrated the computational process of the projected model.

\subsection{PAYOFF SYSTEM}

Joss S anchez Perez [11] has proposed a modification to the concept of the potential of Hart and MasColell to determine a payoff system for job scheduling problems. They obtained explicit formulas for the potential of job scheduling problems and for its corresponding payoff system. Also established a relation between this payoff system and the Shapley value of a certain cooperative game.

\subsection{GENETIC ALGORITHM}

Sayedmohammadreza Vaghefinezhad and Kuan Yew Wong [5] have presented a Genetic Algorithm Approach for Solving a Flexible Job Shop Scheduling Problem .Flexible job shop scheduling had been noticed as an effective manufacturing system to cope with rapid development in today's competitive environment. Flexible job shop scheduling problem (FJSSP) is known as a NP-hard problem in the field of optimization. Considering the dynamic state of the real world makes this problem more and more complicated. Most studies in the field of FJSSP had only focused on minimizing the total makespan. In this, a mathematical model for FJSSP had been developed. The objective function was maximizing the total profit while meeting some constraints. Time-varying raw material costs and selling prices and dissimilar demands for each period, had been considered to decrease gaps between reality and the model. A manufacturer that produced various parts of gas valves had been used as a case study. Its scheduling problem for multi-part, multi-period, and multi-operation with parallel machines had been solved by using genetic algorithm (GA). The best obtained answer determined the economic amount of production by different machines that belonged to predefined operations for each part to satisfy customer demand in each period.

Surekha P and S.Sumathi [12] have presented a genetic algorithm and ant colony optimization algorithm for solving the Job-shop Scheduling Problem (JSSP).The genetic algorithm comprised 
of different stages like generating the initial population, selecting the individuals for reproduction and reproducing new individuals. Ant Colony Optimization (ACO) was a Meta heuristic inspired by the foraging behavior of ants, which was also used to solve this combinatorial optimization problem. In JSSP ants moved from one machine (nest) to another machine (food source) depending upon the job flow, thereby optimizing the sequence of jobs. The sequence of jobs was scheduled using Fuzzy logic and optimizes using GA and ACO. The makespan, completion time, makespan efficiency, algorithmic efficiency and the elapsed time for the genetic algorithm and the ant colony algorithm were evaluated and compared. The improvement in the performance of the algorithms based on the computed parameters was also discussed. Computational results of those optimization algorithms were compared by analyzing the JSSP benchmark instances, FT10 and the ABZ10 problems.

\subsection{JOHNSON'S SEQUENCING RULE}

Punit Kumar Singh and Dr. Rakesh Kumar [13] have presented path optimization algorithm for network problems using job sequencing technique. The job sequencing technique was used to determine an optimal sequence. It performed a series of jobs by a number of specific orders so that it calculated the optimal cost. They proposed a novel approach to find an optimal path from source to destination by taking advantage of job sequencing technique. They had used $\mathrm{n}$ jobs $\mathrm{m}$ machine sequencing technique and this was divided into $\mathrm{n}$ jobs 2 machine problems. Using Johnson's sequencing rule, they solved the problem and obtained the (n-1) sub sequences of the route. Using the proposed algorithm, they calculated the optimal sequence, which lead to the shortest path of the network.

\subsection{MILP APPROACH}

Christodoulos A. Floudas, Xiaoxia Lin [14] have presented the advances of mixed-integer linear programming (MILP) based approaches for the scheduling of chemical processing systems. They focused on the short-term scheduling of general network represented processes. First, the various mathematical models that had been proposed in the literature were classified mainly based on the time representation. Discrete-time and continuous-time models were presented along with their strengths and limitations. Several classes of approaches for improving the computational efficiency in the solution of MILP problems were discussed. Furthermore, a summary of computational experiences and applications wais provided. They concluded with perspectives on future research directions for MILP based process scheduling technologies.

\subsection{SETUP TIME METHOD}

Dr.Khallel Ibrahim Mahmoud and Israr Ahmad [15] have presented Job-Shop Sequencing Real Life Problem with Setup Time. They analyzed the sequencing situations on two machines where the machine setup time was not independent of processing order. A real case study of Hadhramout Industrial Company Complex, Mukalla, Yemen was taken as a model. Data was collected and analyzed using MS-Excel by different methods. The problem formulation had been presented. Multiple solutions were obtained by applying sequencing methods. The comparison of different solutions was done to choose the optimal solution. The time was reduced by $23 \%$ to perform the group of jobs and the setup time was reduced $30.5 \%$ as well as the mean flow time was reduced by $30.5 \%$.

\subsection{MATHEMATICAL MODEL}

Jafar Razmi et al. [16] have proposed a Mathematical Model for a Flow Shop Scheduling Problem with Fuzzy Processing Times. Various methods have been developed by considering 
special conditions. Thus, most of these studies were concentrated on non-exact solutions. In addition, non-deterministic problems were analyzed by fuzzy logic. Some of other studies on stochastic flow shop scheduling problem were also addressed. In this, they considered the fuzzy flow shop scheduling problem. The optimal probability was defined similar to the definition of the optimal index of the stochastic model. The criterion of a better solution selection was the possibility of priority of one sequence to other sequences of the last step. This research had proposed a method to estimate the distribution function of the makespan by using fuzzy numbers and defined the feasibility of the provided solution through this method.

Sequencing is similar to that of scheduling. Job Sequencing is the arrangement of the tasks required to be carried out sequentially. The job sequencing problem can be solved by means of the new modified heuristic technique called time deviation method. The modified time deviation method can be used for processing $n$ jobs through two machines, $n$ jobs through three machines, $n$ jobs through m machines.

\section{MODIFIED TIME DEVIATION METHOD}

Time deviation method is used to obtain the optimal sequence of the jobs. In this method time duration table is calculated for each job in the row wise and the column wise.

1. Maximum time duration of the row minus the time duration of the cell gives the row deviation of the cell in the time duration table.

$$
p_{i j}=r_{i}-t_{i j}
$$

Where $r_{i}$ is the maximum time of the $i^{\text {th }}$ row, $p_{i j}$ is the row time deviation of the $(i, j)^{\text {th }}$ cell and $t_{i j}$ be the time required for processing $i^{\text {th }}$ job on the $j^{\text {th }}$ machine.

2. Maximum time duration of the column minus the time duration of the cell gives the column deviation of the cell in the time duration table.

$$
c_{i j}=s_{i}-t_{i j}
$$

Where $S_{i}$ is the maximum time of the $i^{\text {th }}$ column, $c_{i j}$ is the column time deviation of the $(i, j)^{\text {th }}$ cell and $t_{i j}$ be the time required for processing $i^{\text {th }}$ job on the $j^{\text {th }}$ machine.

The time deviation method used for solving job sequencing problem is explained with the examples.

\subsection{SEQUENCING N JOBS IN TWO MACHINES}

The $\mathrm{N}$ jobs can be sequenced in two machines by the following steps.

1. Calculate the time deviation table for the sequencing problem.

2. The cell which has both the time deviation vectors as zero for machine 1, perform that job first.

3. If more than one cell has both vectors as zero then calculate sum deviation of the corresponding columns. Cell which has largest sum deviation is performed first and so on.

4. Similar steps are followed for machine 2 but here the jobs are performed lastly. 
International Journal of Computer Science \& Information Technology (IJCSIT) Vol 5, No 3, June 2013

5. Again calculate the reduced time deviation table for all non assigned jobs and continue the steps mentioned above.

6. Stop the process if we get sequence involving all jobs.

7. Arrange the obtained sequence in reverse order to get minimum total elapsed time.

Sequencing $\mathrm{N}$ jobs in two machines can be explained by the following example.

Example: Processing of $\mathrm{N}$ jobs in two machines is given as follows

\begin{tabular}{|c|c|c|c|c|c|c|c|c|c|}
\hline Jobs/Machines & J1 & J2 & J3 & J4 & J5 & J6 & J7 & J8 & J9 \\
\hline M1 & 2 & 5 & 4 & 9 & 6 & 8 & 7 & 5 & 4 \\
\hline M2 & 6 & 8 & 7 & 4 & 3 & 9 & 3 & 8 & 11 \\
\hline
\end{tabular}

The time deviation table will be first calculated for the given problem according to the equations (1) and (2). The table is as follows

\begin{tabular}{|l|l|l|l|l|l|l|l|l|l|}
\hline Jobs/Machines & J1 & J2 & J3 & J4 & J5 & J6 & J7 & J8 & J9 \\
\hline M1 & $(7,4)$ & $(4,3)$ & $(5,3)$ & $(0,0)$ & $(3,0)$ & $(1,1)$ & $(2,0)$ & $(4,3)$ & $(5,7)$ \\
\hline M2 & $(5,0)$ & $(3,0)$ & $(4,0)$ & $(7,5)$ & $(8,3)$ & $(2,0)$ & $(8,4)$ & $(3,0)$ & $(0,0)$ \\
\hline
\end{tabular}

The jobs $\mathrm{J} 4$ of machine 1 and $\mathrm{J} 9$ of machine 2 has both vectors zero and is assigned as follows

The reduced time duration table for the remaining jobs is as follows

\begin{tabular}{|c|c|c|c|c|c|c|c|}
\hline Jobs/Machines & J1 & J2 & J3 & J5 & J6 & J7 & J8 \\
\hline M1 & 2 & 5 & 4 & 6 & 8 & 7 & 5 \\
\hline M2 & 6 & 8 & 7 & 3 & 9 & 3 & 8 \\
\hline
\end{tabular}

The time deviation table is calculated for this sequence as follows

\begin{tabular}{|l|l|l|l|l|l|l|l|}
\hline Jobs/Machines & J1 & J2 & J3 & J5 & J6 & J7 & J8 \\
\hline M1 & $(6,4)$ & $(3,3)$ & $(4,3)$ & $(2,0)$ & $(0,1)$ & $(1,0)$ & $(3,3)$ \\
\hline M2 & $(3,0)$ & $(1,0)$ & $(2,0)$ & $(6,3)$ & $(0,0)$ & $(6,4)$ & $(1,0)$ \\
\hline
\end{tabular}

Job J6 in machine 2 has both the vectors as zero and so it is assigned to the sequence as

\begin{tabular}{|l|l|l|l|l|l|l|l|l|}
\hline J4 & & & & & & & J6 & J9 \\
\hline
\end{tabular}

The time duration table for the jobs $\mathrm{J} 1, \mathrm{~J} 2, \mathrm{~J} 3, \mathrm{~J} 5, \mathrm{~J} 7$, and $\mathrm{J} 8$ is specified and the jobs $\mathrm{J} 4$, J6, J9 will not be specified in this table since it is assigned to the machines as shown above.

\begin{tabular}{|l|l|l|l|l|l|l|}
\hline Jobs/Machines & J1 & J2 & J3 & J5 & J7 & J8 \\
\hline M1 & 2 & 5 & 4 & 6 & 7 & 5 \\
\hline M2 & 6 & 8 & 7 & 3 & 3 & 8 \\
\hline
\end{tabular}


The time deviation table for the above jobs is calculated as follows

\begin{tabular}{|l|l|l|l|l|l|l|}
\hline Jobs/Machines & J1 & J2 & J3 & J5 & J7 & J8 \\
\hline M1 & $(5,4)$ & $(2,3)$ & $(3,3)$ & $(1,0)$ & $(0,0)$ & $(2,3)$ \\
\hline M2 & $(2,0)$ & $(0,0)$ & $(1,0)$ & $(5,3)$ & $(5,4)$ & $(0,0)$ \\
\hline
\end{tabular}

The jobs $\mathrm{J} 7$ in machine 1, J2 and $\mathrm{J} 8$ in machine 2 have both vectors zero and it is assigned as follows

\begin{tabular}{|l|l|l|l|l|l|l|l|l|}
\hline J4 & J7 & & & & J2 & J8 & J6 & J9 \\
\hline
\end{tabular}

The time duration table for the remaining jobs $\mathrm{J} 1, \mathrm{~J} 3$, J5 is given as follows

\begin{tabular}{|l|l|l|l|}
\hline Jobs/Machines & J1 & J3 & J5 \\
\hline M1 & 2 & 4 & 6 \\
\hline M2 & 6 & 7 & 3 \\
\hline
\end{tabular}

The time deviation table for the above jobs is calculated as follows

\begin{tabular}{|l|l|l|l|}
\hline Jobs/Machines & J1 & J3 & J5 \\
\hline M1 & $(4,4)$ & $(2,3)$ & $(0,0)$ \\
\hline M2 & $(1,0)$ & $(0,0)$ & $(4,3)$ \\
\hline
\end{tabular}

Here $\mathrm{J} 5$ in machine 1 and $\mathrm{J} 3$ in machine 2 contain both the vectors as zero and so it is assigned and the remaining $\mathrm{J} 1$ is also assigned as follows

\begin{tabular}{|l|l|l|l|l|l|l|l|l|}
\hline J4 & J7 & J5 & J1 & J3 & J2 & J8 & J6 & J9 \\
\hline
\end{tabular}

This sequence is then arranged in the reverse order from last obtained job to the machine 1 jobs and machine 2 jobs. It is represented as follows

\begin{tabular}{|l|l|l|l|l|l|l|l|l|}
\hline J1 & J5 & J7 & J4 & J9 & J6 & J8 & J2 & J3 \\
\hline
\end{tabular}

The above sequence is the required sequence for the given problem. The minimum total elapsed time is calculated for this job sequence. The total elapsed time is given as

Total Elapsed Time $=$ Time between starting the first job in the optimum sequence on machine 1 and completing the last job in the optimum sequence on machine $\mathrm{M}$.

\begin{tabular}{|c|c|c|c|c|}
\hline Job sequence & \multicolumn{2}{|c|}{ Machine 1 } & \multicolumn{2}{c|}{ Machine 2 } \\
& Time-in & Time-out & Time-in & \\
\hline & & 2 & 2 & 8 \\
1 & 0 & 8 & 8 & 11 \\
5 & 2 & 15 & 11 & 14 \\
7 & 8 & 24 & 14 & 18 \\
4 & 15 & 28 & 18 & 29 \\
9 & 24 & 36 & 29 & 38 \\
6 & 28 & 41 & 38 & 46 \\
8 & 36 & & & \\
\hline
\end{tabular}


International Journal of Computer Science \& Information Technology (IJCSIT) Vol 5, No 3, June 2013

\begin{tabular}{|l|l|l|l|l|}
\hline 2 & 41 & 46 & 46 & 54 \\
3 & 46 & 50 & 54 & 61 \\
\hline
\end{tabular}

The minimum total elapsed time thus obtained is 61 hours.

\subsection{SEQUENCING N JOBS IN THREE MACHINES}

The $\mathrm{N}$ jobs can be sequenced in three machines by the following steps

- Calculate the time deviation table for the sequencing problem.

- The cell which has both the time deviation vectors as zero for machine 1 , perform that job first. If both the time deviation vectors are zero in machine 3 then perform that job in the last and if the vectors are zero in machine 2 then find the sum of deviation vectors separately for above and below of the zero cell. Compare both the deviations.

- Perform that particular job first if the sum of deviation vectors above the cell is less than the other one. If both are same then perform the job either in first or last.

- Again calculate the reduced time deviation table for all non assigned jobs and continue the steps mentioned above.

- Stop the process if we get sequence involving all jobs.

- Arrange the obtained sequence in reverse order to get minimum total elapsed time.

Example: $\mathrm{N}$ jobs to be processed in three machines are given as follows

\begin{tabular}{|l|l|l|l|l|l|l|l|}
\hline Jobs/Machines & J1 & J2 & J3 & J4 & J5 & J6 & J7 \\
\hline M1 & 3 & 8 & 7 & 4 & 9 & 8 & 7 \\
\hline M2 & 4 & 3 & 2 & 5 & 1 & 4 & 3 \\
\hline M3 & 6 & 7 & 5 & 11 & 5 & 6 & 12 \\
\hline
\end{tabular}

The time deviation table will be first calculated for the given problem according to the equations (1) and (2). The table is as follows

\begin{tabular}{|l|l|l|l|l|l|l|l|}
\hline Jobs/Machines & J1 & J2 & J3 & J4 & J5 & J6 & J7 \\
\hline M1 & $(6,3)$ & $(1,0)$ & $(2,0)$ & $(5,7)$ & $0,0)$ & $(1,0)$ & $(2,5)$ \\
\hline M2 & $(1,2)$ & $(2,5)$ & $(3,5)$ & $(0,6)$ & $(4,8)$ & $(1,4)$ & $(2,9)$ \\
\hline M3 & $(6,0)$ & $(5,1)$ & $(7,2)$ & $(1,0)$ & $(7,4)$ & $(6,2)$ & $(0,0)$ \\
\hline
\end{tabular}

Jobs $\mathrm{J} 5$ in machine 1 and $\mathrm{J} 7$ in machine 3 have both the vectors zero and so they are assigned to job sequence as follows

\begin{tabular}{|l|l|l|l|l|l|l|}
\hline J5 & & & & & & J7 \\
\hline
\end{tabular}

The time duration table for the jobs other than $\mathrm{J} 5$ and $\mathrm{J} 7$ are given as follows

\begin{tabular}{|l|l|l|l|l|l|}
\hline Jobs/Machines & J1 & J2 & J3 & J4 & J6 \\
\hline M1 & 3 & 8 & 7 & 4 & 8 \\
\hline M2 & 4 & 3 & 2 & 5 & 4 \\
\hline M3 & 6 & 7 & 5 & 11 & 6 \\
\hline
\end{tabular}


International Journal of Computer Science \& Information Technology (IJCSIT) Vol 5, No 3, June 2013

The time deviation table for the above jobs is specified as follows

\begin{tabular}{|l|l|l|l|l|l|}
\hline Jobs/Machines & J1 & J2 & J3 & J4 & J6 \\
\hline M1 & $(5,3)$ & $(0,0)$ & $(1,0)$ & $(4,7)$ & $(0,0)$ \\
\hline M2 & $(1,2)$ & $(2,4)$ & $(3,5)$ & $(0,6)$ & $(1,4)$ \\
\hline M3 & $(5,0)$ & $(4,1)$ & $(6,2)$ & $(0,0)$ & $(5,2)$ \\
\hline
\end{tabular}

The jobs J2, J6 in machine 1 and J4 in machine 3 have both the vectors zero and so they are arranged as follows

\begin{tabular}{|l|l|l|l|l|l|l|}
\hline J5 & J6 & J2 & & & J4 & J7 \\
\hline
\end{tabular}

The time duration table for the remaining jobs is given as follows

\begin{tabular}{|l|l|l|}
\hline Jobs/Machines & J1 & J3 \\
\hline M1 & 3 & 7 \\
\hline M2 & 4 & 2 \\
\hline M3 & 6 & 5 \\
\hline
\end{tabular}

The time deviation table for the above two jobs are given as

\begin{tabular}{|l|l|l|}
\hline Jobs/Machines & J1 & J3 \\
\hline M1 & $(4,3)$ & $(0,0)$ \\
\hline M2 & $(0,2)$ & $(2,5)$ \\
\hline M3 & $(0,0)$ & $(1,2)$ \\
\hline
\end{tabular}

The job $\mathrm{J} 3$ in machine 1 and the job $\mathrm{J} 1$ in machine 3 are then assigned to the sequence as follows

\begin{tabular}{|l|l|l|l|l|l|l|}
\hline J5 & J6 & J2 & J3 & J1 & J4 & J7 \\
\hline
\end{tabular}

This sequence is then arranged in the reverse order from last obtained job to the machine 1 jobs and machine 2 jobs. It is represented as follows

\begin{tabular}{|l|l|l|l|l|l|l|}
\hline J1 & J3 & J2 & J6 & J5 & J7 & J4 \\
\hline
\end{tabular}

The minimum total elapsed time is calculated for the obtained sequence as follows

\begin{tabular}{|c|c|c|c|c|c|c|}
\hline \multirow{2}{*}{$\begin{array}{c}\text { Job } \\
\text { sequence }\end{array}$} & \multicolumn{2}{|c|}{ Machine 1} & \multicolumn{2}{|c|}{ Machine 2} & \multicolumn{2}{|c|}{ Machine 3} \\
\hline & $\begin{array}{l}\text { Time-in } \\
\text { out }\end{array}$ & Time- & $\begin{array}{l}\text { Time-in } \\
\text { out }\end{array}$ & Time- & $\begin{array}{l}\text { Time-in } \\
\text { out }\end{array}$ & Time- \\
\hline 1 & 0 & 3 & 3 & 7 & 7 & 13 \\
\hline 3 & 3 & 10 & 7 & 9 & 13 & 18 \\
\hline 2 & 10 & 18 & 9 & 12 & 18 & 25 \\
\hline 6 & 18 & 26 & 12 & 16 & 25 & 31 \\
\hline 5 & 26 & 35 & 16 & 17 & 31 & 36 \\
\hline 7 & 35 & 42 & 17 & 20 & 36 & 48 \\
\hline 4 & 42 & 46 & 20 & 25 & 48 & 59 \\
\hline
\end{tabular}


Thus the minimum total elapsed time for the given sequence is 59 hours.

\subsection{SEQUENCING N JOBS IN M MACHINES}

The $\mathrm{N}$ jobs can be sequenced in $\mathrm{M}$ machines by the following steps

- The M machine problem is first converted in to the two machine problem by either of the following conditions

Minimum of M1 $\geq$ Maximum of (M2, M3 ... Mm-1)

Minimum of Mm $\geq$ Maximum of (M2, M3 ... Mm-1)

Or both hold.

- The required sequence is then obtained by applying the time deviation method as done in two machine problem previously.

Example: Sequencing of $\mathrm{N}$ jobs in $\mathrm{M}$ machines can be given by the following problem

\begin{tabular}{|l|l|l|l|l|}
\hline Jobs/Machines & J1 & J2 & J3 & J4 \\
\hline M1 & 7 & 6 & 5 & 8 \\
\hline M2 & 5 & 6 & 4 & 3 \\
\hline M3 & 2 & 4 & 5 & 3 \\
\hline M4 & 3 & 5 & 6 & 2 \\
\hline M5 & 9 & 10 & 8 & 6 \\
\hline
\end{tabular}

The $\mathrm{M}$ machine problem is converted into two machine problem by the conditions (3) and (4). The converted two machine problem is given as follows

\begin{tabular}{|l|l|l|l|l|}
\hline Jobs/Machines & J1 & J2 & J3 & J4 \\
\hline G & 17 & 21 & 20 & 16 \\
\hline H & 19 & 25 & 23 & 14 \\
\hline
\end{tabular}

The required job sequence is then obtained by the procedure that is normally applied for the two machine problem as explained by the above example.

\section{Conclusions}

Thus the new modified heuristic technique called time deviation method is used to obtain the required job sequence and the minimum total elapsed time is also calculated for this sequence of jobs by the usual procedure as specified in the above examples. Processing of $\mathrm{N}$ jobs in 2 machines, Processing of $\mathrm{N}$ jobs in 3 machines, Processing of $\mathrm{N}$ jobs in $\mathrm{M}$ machines were also explained by the examples. 


\section{REFERENCES}

[1] Debasis Mishra, Bharath Rangarajan, "Cost Sharing in a Job Scheduling Problem", ACM Conference on Electronic Commerce, 2005.

[2] JacekBtaewicz, Wolfgang Domschke, Erwin Pesch, "The job shop scheduling problem: Conventional and new solution techniques ", European Journal of Operational Research, pp.1-33, 1996.

[3] Nasr Al-Hinai, Tarek Y. ElMekkawy, "Solving the Flexible Job Shop Scheduling Problem with Uniform Processing Time Uncertainty", In Proceedings of World Academy of Science, Engineering and Technology, 2012.

[4] Julia Chuzhoy,Rafail Ostrovsky, Yuval Rabani, "Approximation Algorithms for the Job Interval Selection Problem and Related Scheduling Problems", In proceedings of mathematics of operations research,2006.

[5] Sayedmohammadreza Vaghefinezhad, Kuan Yew Wong, "A Genetic Algorithm Approach for Solving a Flexible Job Shop Scheduling Problem “, International Journal of Computer Science Issues (IJCSI), Vol. 9, Issue 3, May 2012.

[6] Pragati Jain, and Manisha Jain, "Fuzzy TOPSIS Method in Job Sequencing Problems on machines of unequal efficiencies ",Canadian Journal on Computing in Mathematics, Natural Sciences, Engineering and Medicine, Vol. 2, No. 6, June 2011.

[7] S. M. Kamrul Hasan, Ruhul Sarker, and David Cornforth, "GA with Priority Rules for Solving JobShop Scheduling Problems", In proceeding of: Evolutionary Computation, 2008.

[8] Eugeniusz Nowicki and Czesl Aw Smutnicki, "An Advanced Tabu Search Algorithm For The Job Shop Problem", Journal of Scheduling, Vol. 8, pp.145-159, 2005.

[9] Herbert G. Campbell, Richard A. Dudek, and Milton L. Smith, "A Heuristic Algorithm for the N Job, M Machine Sequencing Problem", Journal on Management Science, Vol. 16, No 10, June 1970.

[10] Jean Respen,Nicolas Zufferey,Edoardo Amaldi, "Heuristics for a multi-machine multi-objective job scheduling problem with smoothing costs", Proceeding for the GOL 2012 conference,2012.

[11] Joss Sanchez-Perez, "A Payoff System for Job Scheduling Problems", Journal of Applied Mathematical Sciences, Vol. 5, No. 19, pp.911 - 920, 2011.

[12] Surekha P, S.Sumathi, "Solving Fuzzy based Job Shop Scheduling Problems using Ga and Aco", Journal of Emerging Trends in Computing and Information Sciences, Vol. 1, No. 2, Oct 2010.

[13] Punit Kumar Singh, and Dr. Rakesh Kumar, "Path Optimization Algorithm For Network Problems Using Job Sequencing Technique ",International Journal of Distributed and Parallel Systems (IJDPS), Vol.3, No.3, pp.301-309, May 2012.

[14] Christodoulos A. Floudas,Xiaoxia Lin, "Mixed Integer Linear Programming in Process Scheduling: Modeling, Algorithms, and Applications", Annals of Operations Research, pp. 131-162, 2005.

[15] Dr.Khallel Ibrahim Mahmoud, and Israr Ahmad, "Job Shop Sequencing Real Life Problem With Setup Time ", Journal of Engineering and Technology, Vol.27, No.7, 2009.

[16] Jafar Razmia, Reza Tavakkoli Moghaddam, Mohammad Saffari, "A Mathematical Model for a Flow Shop Scheduling Problem with Fuzzy Processing Times ", Journal of Industrial Engineering, Vol. 3, pp.39-44, 2009. 